Proceedings of SALT 24: 84-101, 2014

\title{
Conditional independence and biscuit conditional questions in Dynamic Semantics*
}

\author{
Katsuhiko Sano \\ Japan Advanced Institute of Science and \\ Technology \\ Yurie Hara \\ City University of Hong Kong
}

Abstract Biscuit conditionals such as If you are thirsty, there's beer in the fridge are felt to be different from canonical conditionals If it's raining, the fireworks will be cancelled in that the consequent seems to be entailed regardless of the truth/falsity of the antecedent. Franke (2009) argues that the "feeling of the consequent entailment" in biscuit conditionals is due to the conditional independence between the antecedent and consequent; thus a uniform semantics for canonical and biscuit conditionals can be maintained. A question arises as to whether it is possible to derive the same consequent entailment in the framework of dynamic semantics. Furthermore, there are some instances of biscuit conditional questions such as If I get thirsty, is there anything in the fridge? This paper provides a dynamic and non-symmetric version of the independence condition, a d-independence condition which correctly derives the consequent entailment in both declaratives and interrogatives.

Keywords: biscuit conditionals, conditional independence, dynamic semantics, questions

\section{Introduction}

Biscuit conditionals such as (1) are felt to be different from canonical conditionals as in (2) in that the consequent seems to be entailed regardless of the truth of the antecedent.

\section{(1) Biscuit Conditional}

a. If you are thirsty, there's beer in the fridge.

ENTAILS

There's beer in the fridge.

* We thank Makoto Kanazawa, Stefan Kaufmann, the anonymous reviewers of SALT24, the audience at SALT24 at NYU and Semantic Research Group at Keio University for their insightful comments. We are also grateful to Todd Snider for his work on the editorial matter. All remaining errors are our own. The work of the first author was partially supported by JSPS KAKENHI, Grant-in-Aid for Young Scientists (B) 24700146.

(C)2014 Sano and Hara 
Conditional independence and biscuit conditional questions

b. There are biscuits on the sideboard if you want them.

ENTAILS

There are biscuits on the sideboard.

(2) Canonical Conditional

If it's raining, the fireworks will be cancelled.

DOESN'T ENTAIL

The fireworks will be cancelled.

In other words, biscuit conditionals do not give rise to 'conditional' meanings. As noted by Iatridou (1991: 51), the if-clauses in canonical conditionals restrict the situations where the consequent is true, while those in biscuit conditionals restrict the situations where "the consequent is relevant (in a vague sense, also subsuming circumstances of social appropriateness)."

Given this difference, various scholars attempted to propose a special semantics for biscuit conditionals. For instance, Siegel (2006) proposes that a biscuit conditional construction gives rise to existential quantification over potential literal acts. Simply put, (1a) is paraphrased as in (3).

(3) If you're thirsty, there is a (relevant/salient) assertion that there's beer in the fridge.

There are a number of problems in this approach. In particular, Siegel's paraphrase asserts a mere presentation of relevant potential act, which is too weak. The point might be clearer with a biscuit conditional question like (4). In uttering (4), the speaker is waiting for the addressee's answer, thus performing an actual question act rather than merely presenting a relevant possible act as in (5).

(4) If I get thirsty, is there beer in the fridge?

(5) If I get thirsty, there is a relevant question: Is there beer in the fridge?

Franke (2009), on the other hand, argues that no special semantics for biscuit conditionals are necessary. The conditional independence derives the consequent entailment of biscuit conditionals. Assuming that Franke (2009) is correct, a question arises as to whether it is possible to derive the same consequent entailment in the framework of dynamic semantics. Furthermore, there are some instances of biscuit conditional questions, as in (4).

Intuitively, a biscuit conditional question does give rise to a consequent entailment. In (4), answering 'yes' entails that there is something in the fridge and answering 'no' entails the opposite regardless of the state of the speaker's thirst. Put another way, if the speaker asks the unconditionalized counterpart right after the conditionalized one, it would be a superfluous question. In contrast, canonical 
conditional questions do not. I.e., answering 'yes' to (6) does not enlighten the questioner on whether the fireworks will be cancelled or not when it is not raining.

(6) If it's raining, will the fireworks be cancelled?

DOESN'T ENTAIL

Will the fireworks be cancelled?

This paper provides a dynamic and non-symmetric version of the independence condition, a $d$-independence condition which correctly derives the consequent entailment in both declaratives and interrogatives. ${ }^{1}$

We would like to note that this paper does not attempt to specify a sufficient condition for the biscuit conditional. That is, we do not argue that independence alone gives us a felicitous biscuit conditional. For instance, although it is a reasonable assumption that the antecedent and consequent in (7) are independent, the whole conditional is not a felicitous biscuit conditional.

\#If France is hexagonal, there's a beer in the fridge.

Thus, independence is one of the necessary conditions for the biscuit conditional interpretation but not a sufficient condition. In order to be a felicitous biscuit conditional, the information encoded in the antecedent must be relevant to the information encoded in the consequent. Note also that there are arguably a lot of ways to come up with a context which makes the antecedent and consequent in (7) relevant. In those cases, (7) might be judged as a felicitous biscuit conditional. We refer the interested reader to Franke 2007, 2009 for the discourse functions of biscuit conditionals.

This paper is structured as follows: First, we review Franke's $(2007,2009)$ definition of independence and his analysis of biscuit conditionals in static semantics in section 2. Crucially, the independence assumption enables us to have a uniform semantics for both canonical and biscuit conditionals. Section 3.1 motivates the dynamic extension. Dynamic semantics is suitable for analyzing biscuit conditionals since intuitively the antecedent of a biscuit conditional sets up a context for the subsequent update, i.e., assertion or question. Section 3.2 then introduces our definition of $d$-independence, and show how it derives the consequent entailment in Kaufmann's (2000) dynamic framework. In section 4, we turn to the analysis of biscuit conditional questions like (4) by further extending the notion of independence to a structured context (Groenendijk 1999). In particular, we adopt Isaacs and Rawlins' (2008) analysis of conditional questions which combines Kaufmann's (2000) stack-based model of conditionals and Groenendijk's (1999) theory of interrogatives. Together with our $d$-independence condition, the consequent entailment of a biscuit conditional question is successfully derived. Section 5 concludes the paper.

1 Incidentally, van Rooij (2007) mentions a condition equivalent to our $d$-independence. See Remark 6. 


\section{Independence and biscuit conditionals and in static semantics}

Franke (2009) argues that the "feeling of the consequent entailment" in biscuit conditionals is due to the conditional independence between the antecedent and consequent; thus a uniform semantics (i.e., a strict implication) for canonical and biscuit conditionals can be maintained. ${ }^{2}$

Let us go back to the biscuit conditionals and conditional independence. Given a set $W$ of possible worlds, we regard a set $\sigma \subseteq W$ as the speaker's epistemic state. Let $P, Q \subseteq W$ be propositions. We denote the complement of $P$ over $W$ by $\bar{P}$. Following Franke (2009), we introduce the following definitions.

a. The speaker knows the proposition $P$ ( $\square P$, in short) in $\sigma$ if $\sigma \subseteq P$.

b. $P$ is consistent $(\diamond P$, in short) in $\sigma$ if $\sigma \cap P \neq \emptyset$.

c. 'if $P$ then $Q$ ' holds in $\sigma$ if $\sigma \cap P \subseteq Q$.

Note that the semantics of implication above is identical to that of strict implication. Franke (2009) shows that the consequent entailment $\square Q$ follows from a strict implication 'if $P$ then $Q$ ', together with the following independence assumption.

Definition 1. Let $\sigma \subseteq W$ be an epistemic state and $P, Q \subseteq W$ propositions. We say that $P$ and $Q$ are independent in $\sigma$ if

$$
\diamond X \text { and } \diamond Y \text { in } \sigma \text { imply } \diamond(X \cap Y) \text { in } \sigma,
$$

for all $X \in\{P, \bar{P}\}$ and $Y \in\{Q, \bar{Q}\}$.

Let us take Figure 1 for illustration, where $p$ and $q$ are the syntactic expressions of sets $P$ and $Q$, respectively. We define our set of possible worlds as all the truth functions from $\{p, q\}$ to the set $\{0,1\}$ of truth values. Each circle in Figure 1 represents a world and orange ellipses represent an information state $\sigma_{i}(i=1$ or 2). In the current setting, our $W$ only contains four worlds. The world $w_{11}$, for instance, represents a truth function sending both $p$ and $q$ to 1 and the world $w_{10}$ is a truth function sending $p$ to 1 and $q$ to 0 . We can show that $P$ and $Q$ are independent in $\sigma_{1}$

2 As noted by van Rooij (2007) and Franke (2009), the notion of independence is deployed in various areas. Let us take a brief look at some of them. The first one is logical independence. Given an axiom system $S$, a proposition $A$ is independent of $S$ if neither $A$ nor $\neg A$ is derivable from $S$. The second one is probabilistic independence. Given a provability function $P$, we say that a proposition $A$ is probabilistically independent from a proposition $B$ if $P(A \wedge B)=P(A) \cdot P(B)$, or equivalently, if $P(A) \neq 0$, then $P(B \mid A)=P(B)$. Third, Lewis (1988) defines the notion of orthogonality of subject matter (i.e., questions/issues): For a context $\sigma$ (a set of possible worlds): Two issues (i.e., equivalence relations on $\sigma) C_{1}$ and $C_{2}$ are independent/orthogonal in $\sigma$ iff, for all $w, v \in \sigma$, there exists $u \in \sigma$ such that $\langle u, w\rangle \in C_{1}$ and $\langle u, v\rangle \in C_{2}$. Our notion of independence in dynamic semantics is similar to the second definition of probabilistic independence: $P(A) \neq 0$, then $P(B \mid A)=P(B)$. 


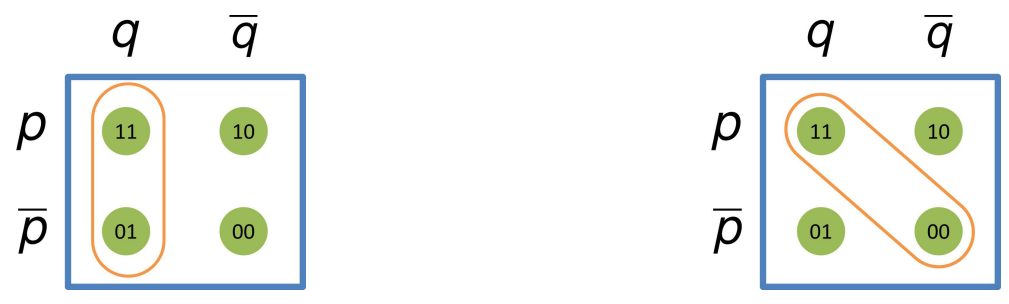

$\begin{array}{ll}\text { (i) } p \text { and } q \text { are independent in } \sigma_{1} & \text { (ii) } p \text { and } q \text { are not independent in } \sigma_{2}\end{array}$

Figure 1 (In)dependence in static semantics

in Figure 1 (i) as follows: First, as for the case where $X=P$ and $Y=Q$, we have both $\diamond P$ and $\diamond Q$ in $\sigma_{1}$ and $\diamond(P \cap Q)$ in $\sigma_{1}$. Second, when $X=\bar{P}$ and $Y=Q$, we also have both $\diamond \bar{P}$ and $\diamond Q$ in $\sigma_{1}$ and $\diamond(\bar{P} \cap Q)$ in $\sigma_{1}$. Finally, as for the cases where $Y=\bar{Q}, \bar{Q}$ is not consistent in $\sigma_{1}$, thus the antecedent in Definition 1 is trivially met. In contrast, $P$ and $Q$ are not independent in $\sigma_{2}$ of Figure 1 (ii), since $\diamond \bar{P}$ and $\diamond Q$ in $\sigma_{2}$ but $\bar{P} \cap Q$ is not consistent in $\sigma_{2}$.

We can characterize the notion of independence in terms of $\square$ as follows.

Proposition 2. Let $\sigma \subseteq W$ be an epistemic state and $P, Q \subseteq W$ propositions. Then, the following are equivalent:

(i) $P$ and $Q$ are independent in $\sigma$,

(ii) if $X$ is consistent in $\sigma$, then

$\square Y$ in $\sigma$ is equivalent to $\square Y$ in $\sigma \cap X$,

for all $X \in\{P, \bar{P}\}$ and $Y \in\{Q, \bar{Q}\}$.

Proof. (i) is equivalent to the following: if $X$ is consistent in $\sigma, \diamond Y$ in $\sigma$ is equivalent to $\diamond Y$ in $\sigma \cap X$, for all $X \in\{P, \bar{P}\}$ and $Y \in\{Q, \bar{Q}\}$. Moreover, this is equivalent to the following: if $X$ is consistent in $\sigma, \square \bar{Y}$ in $\sigma$ is equivalent to $\square \bar{Y}$ in $\sigma \cap X$, for all $X \in\{P, \bar{P}\}$ and $Y \in\{Q, \bar{Q}\}$. Then, it is easy to see that this is equivalent to (ii).

Now let us derive the consequent entailment from the conditional independent assumption.

Proposition 3 (Franke 2009). Let $\sigma \subseteq W$ be an epistemic state and $P, Q \subseteq W$ propositions. Suppose that $P$ and $Q$ are independent in $\sigma$ and that $P$ is consistent in $\sigma$. Then, 'if $P$ then $Q$ ' in $\sigma$ implies $\square Q$ in $\sigma$.

Briefly, if the antecedent of a conditional is presupposed to be possible ( $\diamond P$ in $\sigma)$ and the speaker has a prior knowledge that the antecedent $P$ and the consequent 
Conditional independence and biscuit conditional questions

$Q$ are independent (Definition 1), it follows from $\sigma \cap P \subseteq Q$ that the speaker knows $Q$, hence the entailment of the consequent obtains.

Proof. Assume that 'if $P$ then $Q$ ' in $\sigma$, i.e., $\sigma \cap P \subseteq Q$ hence $\square Q$ in $\sigma \cap P$. By assumption and Proposition 2, we obtain $\square Q$ in $\sigma$, as desired.

In short, a strict implication with the independence assumption gives us the consequent entailment. Thus, a uniform semantics, i.e., strict implication, for canonical and biscuit conditionals can be maintained and no special semantics for biscuit conditionals is necessary.

\section{Dynamic extension}

\subsection{Why dynamic?}

The previous section summarized Franke's (2009) static analysis that derives the consequent entailment of biscuit conditionals. This paper extends the same idea to dynamic framework. In particular, we adopt procedural/suppositional semantics of conditionals. Within the dynamic view, conditionals are characterized as a two-step (Stalnaker 1968; Karttunen 1974; Heim 1982) or three-step (Kaufmann 2000; Isaacs \& Rawlins 2008) update procedure:

(i) A temporary state is created by updating the information state with the antecedent of the conditional.

(ii) The derived state is updated with the consequent.

(iii) The original context learns the effects of the second step.

It is unclear if there is any motivation/advantage of the dynamic approach. There are two clear advantages to this approach. First, as Franke (2007) notes, one of the discourse functions of a biscuit conditional is that its antecedent shifts the context so that the subsequent assertion becomes optimal. For instance, in (1a), the consequent clause there's beer in the fridge "is possibly irrelevant, infelicitous or in some other sense non-optimal" (Franke 2007: 96) in the original unmodified context. Thus, the antecedent clause if you're thirsty shifts the context so that the consequent can be felicitously uttered. The dynamic approach of conditionals fits this intuition. Stalnaker (1968) summarizes the step-wise semantics of conditionals, which is coined "the Ramsey test":

"First add the antecedent (hypothetically) to your stock of beliefs; second, make whatever adjustments are required to maintain consistency (without modifying the belief in the antecedent); finally, 
consider whether or not the consequent is then true." (Stalnaker 1968: 102)

Second, as argued by Isaacs \& Rawlins (2008), a dynamic semantics of conditionals is suitable for accounting conditional questions. One of the main goals of this paper is to account for the consequent entailment of biscuit conditional questions without stipulating special semantics for them. Conditional questions can also have procedural/suppositional semantics as noted by Ramsey (1931):

"If two people are arguing 'If $p$, will $q$ ?' and are both in doubt as to $p$, they are adding $p$ hypothetically to their stock of knowledge and arguing on that basis about $q$..." (Ramsey 1931: 247)

Isaacs \& Rawlins (2008) argue for this dynamic and suppositional theory of conditionals and develop the formal apparatus as detailed in section 4 of this paper. In particular, Isaacs \& Rawlins (2008) argue against Velissaratou's (2000) static logic of conditional questions, which regards denial of the antecedent like (9b) as a partial answer to the conditional question.

(9) a. If Alfonso comes to the party, will Joanna leave?

b. Alfonso isn't coming to the party.

(Isaacs \& Rawlins 2008)

According to Isaacs \& Rawlins (2008), Velissaratou's (2000) approach is undesirable in both empirical and conceptual senses. Empirically, denial of the antecedent does not answer the question but only dispels the issue. Conceptually, including denial of the antecedent as an answer to the question results in abandoning the mutual exclusivity property of questionhood (Hamblin 1958). See Isaacs \& Rawlins 2008 for more detailed discussions.

Also, we note that the dynamic turn makes a difference with the semantics of might (see Remark 7 and Footnote 6 below).

\subsection{Independence and biscuit conditionals in dynamic semantics}

In the current paper, we follow Kaufmann's (2000) formulation of dynamic semantics. First, we regard a mapping from a countable set $\mathrm{P}$ of proposition letters to $\{0,1\}$ as a possible world, define $W:=\{0,1\}^{\mathrm{P}}$ as the set of all possible worlds and say that $\sigma \subseteq W$ is an information state. We assume that our syntax $\mathscr{M} \mathscr{L}$ consists of the negation $\neg$, the conjunction $\wedge$, the implication $\rightarrow$, and the diamond operator $\diamond$ ('might' as a test, for more details, see Remark 7 below), as well as P. The formulas in $\mathscr{M} \mathscr{L}$ are defined inductively as usual. Then, we define the result of updating $\sigma$ 
Conditional independence and biscuit conditional questions

with a formula $\varphi \in \mathscr{M} \mathscr{L}$ as follows:

$$
\begin{array}{ll}
\sigma[p] & =\{w \in \sigma \mid w(p)=1\} \\
\sigma[\varphi \wedge \psi] & =\sigma[\varphi][\psi], \\
\sigma[\neg \varphi] & =\sigma \backslash \sigma[\varphi], \\
\sigma[\varphi \rightarrow \psi] & =\{w \in \sigma \mid w \in \sigma[\varphi] \text { implies } w \in \sigma[\varphi][\psi]\} \\
\sigma[\diamond \varphi] & =\{w \in \sigma \mid \sigma[\varphi] \neq \emptyset\} .
\end{array}
$$

In characterizing the intuition of entailment, we use the notion of support (acceptance in Veltman 1996) and consistency in an information state:

(10) $\varphi$ is supported in $\sigma$ (notation: $\sigma \models \varphi$ ) if $\sigma[\varphi]=\sigma$.

(11) $\varphi$ is consistent in $\sigma$ if $\sigma[\varphi] \neq \emptyset$.

In Kaufmann 2000, note that we obtain the monotonicity of the updates in the following sense: ${ }^{3}$

Proposition 4. $\sigma[\varphi] \subseteq \sigma$ for all information states $\sigma$ and all formulas $\varphi$.

We define the non-symmetric $d$-independent condition as in Definition 5. Intuitively speaking, $\psi$ is independent of $\varphi$ in $\sigma$ if updating $\sigma$ with $\varphi$ or $\neg \varphi$ does not affect the consistency of $\psi$.

Definition 5. $\psi$ is $d$-independent of $\varphi$ in $\sigma$ if, given $X$ 's consistency in $\sigma$,

$$
\sigma[Y] \neq \emptyset \text { is equivalent to } \sigma[X][Y] \neq \emptyset,
$$

for all $X \in\{\varphi, \neg \varphi\}$ and all $Y \in\{\psi, \neg \psi\}$.

Remark 6. Van Rooij (2007) defines a similar yet different notion of independence which follows Lewis's (1988) idea of orthogonality, as follows: We say that $\varphi$ and $\psi$ are independent in $\sigma$ if the partitions $\{\sigma[\varphi], \sigma[\neg \varphi]\}$ and $\{\sigma[\psi], \sigma[\neg \psi]\}$ are orthogonal, i.e., for all $X \in\{\sigma[\varphi], \sigma[\neg \varphi]\}$ and $Y \in\{\sigma[\psi], \sigma[\neg \psi]\}, X \cap Y \neq \emptyset$. Note that this definition is symmetrically defined for $\varphi$ and $\psi$. Van Rooij (2007) also mentions a condition, his Lemma 4.3 (independence), which is equivalent to

3 One of the differences between Kaufmann (2000) and Veltman (1996) lies in the semantics for $\diamond \varphi$. If we follow the semantics of $\diamond \varphi$ in Kaufmann 2000, all the updates are monotone: $\sigma[\varphi] \subseteq \sigma$ for all $\sigma$ and $\varphi$. In Veltman 1996, however, we have the following semantics for $\diamond \varphi$ :

$$
\sigma[\diamond \varphi]=\{w \in W \mid \sigma[\varphi] \neq \emptyset\} .
$$

As a result, all the updates need not to be monotone. In this sense, $\diamond$ does not maintain monotonicity in updates in Veltman 1996. Note that monotonicity of updates are essential in characterizing the independence and consequent entailment of biscuit conditionals. 
our Definition 5. However, van Rooij's syntax does not include the test modality $\diamond$. Thus, once we include $\diamond$ in the syntax, the two versions are no longer equivalent. For more details, see Footnote 5.

a. $q$ is $d$-independent of $p$ in $\sigma_{1}$ of Figure 1 (i). This is shown as follows. First, let $X$ be $p$. When $Y$ is $\neg q$, we obtain that $\neg q$ is inconsistent in both $\sigma_{1}$ and $\sigma_{1}[p]$. When $Y$ is $q, \sigma_{1}[q]=\left\{w_{11}, w_{01}\right\}$ and $\sigma_{1}[p][q]=\left\{w_{11}\right\}$, and so $q$ is consistent in both $\sigma_{1}$ and $\sigma_{1}[p]$. Second, let $X$ be $\neg p$. Similarly to the first case, when $Y$ is $\neg q$, we obtain that $\neg q$ is inconsistent in both $\sigma_{1}$ and $\sigma_{1}[p]$. When $Y$ is $q, \sigma_{1}[q]=\left\{w_{11}, w_{01}\right\}$ and $\sigma_{1}[\neg p][q]=\left\{w_{01}\right\}$, and so $q$ is consistent in both $\sigma_{1}$ and $\sigma_{1}[p]$.

b. $q$ is not $d$-independent of $p$ in $\sigma_{2}$ of Figure 1 (ii). Take $\neg p$ as $X$ and $q$ as $Y$ in Definition 5. Clearly, $\neg p$ is consistent in $\sigma_{2}$ because $\sigma_{2}[\neg p]=\left\{w_{00}\right\}=$ $\neq \emptyset$. On the other hand, $\sigma_{2}[q]=\left\{w_{11}\right\} \neq \emptyset$ but $\sigma_{2}[\neg p][q]=\emptyset$.

c. $\diamond p$ is not $d$-independent of $p$ in $\sigma_{2}$ of Figure 1 (ii). To see this, take $\neg p$ as $X$ and $\diamond p$ as $Y$ in Definition 5. As in the previous item, $\neg p$ is consistent in $\sigma_{2}$. Then, $\sigma_{2}[\diamond p]=\sigma_{2} \neq \emptyset$ while $\sigma_{2}[\neg p][\diamond p]=\emptyset$.

d. $p$ is $d$-independent of $\diamond p$ in $\sigma_{2}$ of Figure 1 (ii). When we take $\neg \diamond p$ as $X, \sigma_{2}[\neg \diamond p]=\emptyset$, and so, we can focus on the case where $X$ is $\diamond p$. Now, $\sigma_{2}[\diamond p]=\sigma_{2} \neq \emptyset$. For any $Y \in\{p, \neg p\}$, thus, we obtain $Y$ 's consistency in $\sigma_{2}$ hence $Y$ 's consistency in $\sigma_{2}[\diamond p]$.

As (12c) and (12d) show, our independence condition is non-symmetric, i.e., only defines the consequent's independence from the antecedent, since in the current analysis, a conditional is treated as a procedural update. ${ }^{4}$ This non-symmetry is particularly suitable for the biscuit conditional questions discussed below, as the antecedent assertion sets up a context on which the consequent question operate. Van Rooij (2007) also offers a notion of independence in context in a dynamic setting to account for the strengthening of conditional presuppositions, but it is symmetrically defined (van Rooij 2007: Definition 4.2). ${ }^{5}$

Remark 7. As noted above in section 3.1, the treatment of might is one of the crucial differences between static and dynamic semantics. In static semantics, might is defined as existential quantification over possible worlds (e.g., Kratzer 1981, 1991). In dynamic semantics, might $p$ (denoted by $\diamond p$ in our syntax) is a test on $\sigma$

4 In (12), note that we can also show $p$ is $d$-independent of $q$ in both $\sigma_{1}$ and $\sigma_{2}$. Therefore, our notion of $d$-independence is symmetric for $p$ and $q$.

5 As we have seen in (12), $\diamond$ is the source of non-symmetry of $d$-independence in our case. In particular, it is easy to see that $\diamond p$ and $p$ are not independent in $\sigma_{2}$ of Figure 1 by van Rooij's (2007) definition (see Remark 6), while $p$ is $d$-independent of $\diamond p$ in $\sigma_{2}$. 
as defined above, thus it does not bring any new information on $\sigma$. Veltman (1996) argues that this dynamic semantics of might is also one of the advantages over the static one since it captures the difference in the following pair:

a. Somebody is knocking at the door $\left(p_{1}\right) \ldots$ Maybe it's John $\left(\diamond p_{2}\right) \ldots$ It's Mary $\left(p_{3}\right)$.

b. \# Somebody is knocking at the door $\left(p_{1}\right) \ldots$ Maybe it's John $\left(\diamond p_{2}\right) \ldots$ It's Mary $\left(p_{3}\right) \ldots$ Maybe it's John $\left(\diamond p_{2}\right)$. (adapted from Veltman 1996: 223)

In (13a), the speaker's presumption is conceivably overridden by the learned fact. Since $\diamond p_{2}$ is a mere test, $\sigma\left[p_{1}\right]\left[\diamond p_{2}\right]=\sigma\left[p_{1}\right]$, if $p_{2}$ is consistent in $\sigma\left[p_{1}\right]$. Thus, $\sigma\left[p_{1}\right]\left[\diamond p_{2}\right]\left[p_{3}\right]$ does not lead to an absurd state, if $p_{3}$ is consistent in $\sigma\left[p_{1}\right]\left[\diamond p_{2}\right]$. In (13b), on the other hand, the speaker cannot come up with a new assumption which overrides the established fact. Once $\sigma\left[p_{1}\right]\left[\diamond p_{2}\right]$ is updated with $p_{3}, p_{2}$ is no longer consistent (assuming only one person can knock at the door), thus $\sigma\left[p_{1}\right]\left[\diamond p_{2}\right]\left[p_{3}\right]\left[\diamond p_{2}\right]$ results in an absurd state. ${ }^{6}$

Going back to the definition of $d$-independence, since the negation is defined as $\sigma[\neg \varphi]=\sigma \backslash \sigma[\varphi]$, a simple calculation shows that $\sigma[\neg \varphi]=\emptyset$ iff $\sigma[\varphi]=\sigma$. With the help of this equivalence, we can rewrite the $d$-independence in terms of the notion of support as follows.

Proposition 8. $\psi$ is $d$-independent of $\varphi$ in $\sigma$ iff, given $X$ 's consistency in $\sigma$,

$$
\sigma \mid=Y \text { is equivalent to } \sigma[X] \models Y \text {, }
$$

for all $X \in\{\varphi, \neg \varphi\}$ and all $Y \in\{\psi, \neg \psi\}$.

6 Since the interest of the current paper lies in conditionals, the reader might wonder what the dynamic approach predicts for the conditional sentences which contain $p$ and $\diamond p$. According to (12c) and (12d), $\diamond p$ is not $d$-independent of $p$ while $p$ is $d$-independent of $\diamond p$. Thus, we predict that $p \rightarrow \diamond p$ does not entail $p$, while $\diamond p \rightarrow p$ entails $p$. We believe that this prediction is correct, although the judgment of the natural language constructions turns out to be somehow more intriguing as in (i).

(i) a. \# If it's raining, it might be raining.

b. If it might be raining, it's raining.

If the independence were the sufficient condition for biscuit conditionals, (ia) would be a canonical conditional, while (ib) would be a biscuit conditional. However, the contrast of felicity in (i) does not seem to come from their biscuit-conditional-ness. As noted in section 1, independence alone does not yield a biscuit conditional. We speculate that the anomaly of (ia) is similar to Veltman's (13b). The antecedent creates a hypothetical context where 'it's raining' is already a fact. It does not make sense to weaken the established fact with might. Also, it is not clear whether (ib) is a biscuit conditional even though it entails the consequent. Our speculation is that a conditional construction is understood as a biscuit conditional when the antecedent serves as an optimizer for the subsequent speech act (Franke 2007). 
Finally, we obtain the following theorem that derives the consequent entailment of biscuit conditionals. Assume that the consequent is $d$-independent of the antecedent and the antecedent is presupposed to be consistent. Then, when the implication is supported, the consequent alone is also supported:

Theorem 1. Let $\psi$ be $d$-independent of $\varphi$ in $\sigma$ and assume that $\varphi$ is consistent in $\sigma$. Then, $\sigma \models \varphi \rightarrow \psi$ implies $\sigma \models \psi$.

Proof. Assume $\sigma[\varphi] \neq \emptyset$ and $\sigma=\varphi \rightarrow \psi$. By Proposition 8, it suffices to show $\sigma[\varphi][\psi]=\sigma[\varphi]$, i.e., $\sigma[\varphi] \subseteq \sigma[\varphi][\psi]$ by monotonicity. Fix any $w \in \sigma[\varphi]$. Since $\sigma[\varphi \rightarrow \psi]=\sigma, w \in \sigma[\varphi \rightarrow \psi]$. By $w \in \sigma[\varphi], w \in \sigma[\varphi][\psi]$, as desired.

Let us take (1a) as an example. Assume a normal (i.e., non-magical) situation where acquiring the knowledge that the addressee is thirsty does not determine whether there is beer in the fridge or not. Thus, the proposition 'there's beer in the fridge' is independent of 'you are thirsty'. Now, the speaker uttered the sentence (1a). Given the $d$-independence condition and Theorem 1, the consequent proposition 'there's beer in the fridge' is supported. Thus, our condition derives the consequent entailment in the dynamic framework.

Remark 9. As stressed in section 3.1, our semantics of conditionals is procedural and stepwise. Kaufmann (2000) reinforces this thesis by reformulating the semantics of conditionals in terms of the notion of 'conclude'. More specifically, the final step of the conditional update is performed by the operation of 'learning in a state $\sigma$ that a state $\sigma^{\prime}$ supports $\varphi^{\prime}$ (notation: $\sigma\left[\sigma^{\prime} \vdash \varphi\right]$ in our setting). Furthermore, his semantic framework centers around a stack model. To see this, we define $\sigma\left[\sigma^{\prime} \vdash \varphi\right]$ as the following:

$$
\sigma\left[\sigma^{\prime} \vdash \varphi\right]:=\left\{w \in \sigma \mid w \in \sigma^{\prime} \text { implies } w \in \sigma^{\prime}[\varphi]\right\} .
$$

As can be seen, $\sigma[\varphi \rightarrow \psi]=\sigma[\sigma[\varphi] \vdash \psi]$. Thus, the update performed by a conditional $\varphi \rightarrow \psi$ is paraphrased as 'learning in a state $\sigma$ that a state $\sigma[\varphi]$ supports $\psi^{\prime}$. In the next section, we consider the notion corresponding to $\sigma\left[\sigma^{\prime} \vdash \varphi\right]$ in partition semantics. In particular, we extract dynamic semantics for conditional assertions and conditional questions from the work by Isaacs \& Rawlins (2008).

\section{Biscuit conditional questions}

As noted in section 1, there are instances of biscuit conditional questions as in (4), repeated here as (14). Unlike a canonical conditional question like (6), repeated here as (15), (14) does entail the question without if-clause, 'Is there beer in the fridge?'.

(14) If I get thirsty, is there beer in the fridge? 
Conditional independence and biscuit conditional questions

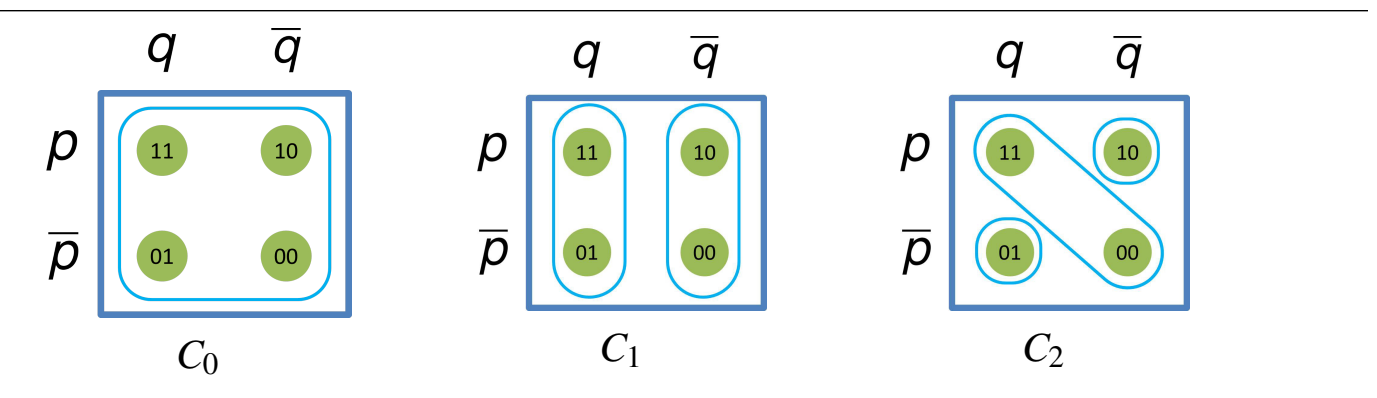

Figure 2 Structured contexts

(15) If it's raining, will the fireworks be cancelled?

We extend our dynamic independence to structured contexts to handle biscuit conditional questions. As before, we stipulate $W:=\{0,1\}^{P}$, where $P$ is the set of proposition letters. In dealing with statements and questions, we now introduce a structured context $C$ as an equivalence relation on some set of possible worlds (Groenendijk 1999; Isaacs \& Rawlins 2008). Each equivalence relation on $W$ gives us a partition as in Figure 2, where blue lines represent equivalence relations. For example, all four worlds in the structured context $C_{0}$ of Figure 2 are indistinguishable, thus the conversation agents are not aware of the difference from each other. For the structured context $C_{1}$ in Figure 2, $q$-worlds are distinguished from $\neg q$-worlds but the agents cannot distinguish, for instance, a $p$-world $w_{11}$ from another $p$-world $w_{01}$. We define the set $\operatorname{Bool}(P)$ as all the propositional combinations generated from $P$. Note that we can calculate the truth value of $w(\varphi)$ for a $w \in W$ and $\varphi \in \operatorname{Bool}(\mathrm{P})$. Now, we define the set $\mathscr{Q} \mathscr{L}$ of query-formulas (see Velissaratou 2000) by:

$$
\text { If } \varphi, \psi \in \operatorname{Bool}(\mathrm{P}) \text { then } \varphi !, \varphi ?, \varphi ! \rightarrow \psi !, \varphi ! \rightarrow \psi ? \text { are in } \mathscr{Q} \mathscr{L} \text {. }
$$

Here, $\varphi$ ! and $\varphi$ ? represent declarative and interrogative sentences, respectively. Moreover, we regard $\varphi ! \rightarrow \psi !, \varphi ! \rightarrow \psi$ ? as a declarative conditional and a conditional question, respectively. We denote query-formulas of $\mathscr{Q} \mathscr{L}$ by $\alpha, \beta$, $\gamma$, etc. We employ the syntactic notion of query-formula from Velissaratou (2000), and follow the central idea of Isaacs \& Rawlins (2008), but our semantics is different from the one defined by Velissaratou (2000). That is, we define the result of updating $C$ with a query-formula of $\mathscr{Q} \mathscr{L}$ as follows:

$$
\begin{aligned}
& C[\varphi !]:=\{\langle w, v\rangle \in C \mid w(\varphi)=v(\varphi)=1\} \\
& C[\varphi ?]:=\{\langle w, v\rangle \in C \mid w(\varphi)=v(\varphi)\} .
\end{aligned}
$$

Let us illustrate some simple assertive and inquisitive updates with figures. $C_{2}$ represents an inquisitive state where the agent does not distinguish $w_{11}$ from $w_{00}$, as 


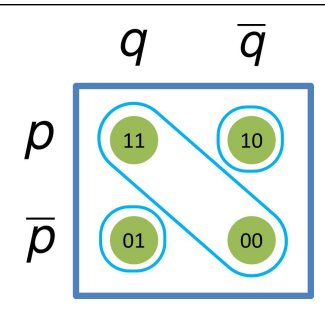

(i) $C_{2}$

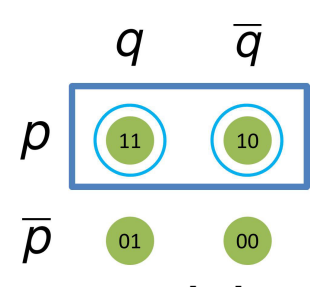

(ii) $C_{2}[p !]$

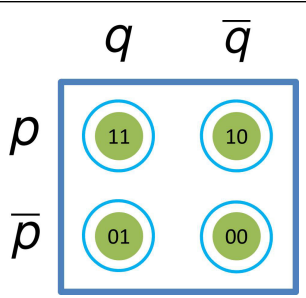

(iii) $C_{2}[q ?]$

Figure $3 \quad q$ ? is not $d$-independent from $p$ ! in $C_{2}$

depicted in Figure 3 (i). In Figure 3 (ii), $C_{2}$ is assertively updated with $p$, thus the worlds that make $p$ false are removed. In Figure 3 (iii), $C_{2}$ is inquisitively updated with $q$ and the worlds that assign different truth values to $q$, i.e., $w_{11}$ and $w_{00}$, are disconnected.

Now, based on Isaacs \& Rawlins (2008), we define conditional updates as follows:

Definition 10. Let $\gamma$ be one of a declarative $\psi$ ! or an interrogative $\psi$ ?. Then,

$$
\begin{aligned}
C[\varphi ! \rightarrow \gamma] & :=C[\neg \varphi !] \cup C[\varphi !][\gamma] \\
& =\{\langle w, v\rangle \in C \mid\langle w, v\rangle \in C[\neg \varphi !] \text { or }\langle w, v\rangle \in C[\varphi !][\gamma]\}
\end{aligned}
$$

Remark 11. Isaacs \& Rawlins (2008) incorporate Kaufmann's (2000) stack model inside the definition of conditionals and conditional questions (see Remark 9). Instead, our Definition 10 above is defined directly against a structured context. Let us see whether our Definition 10 still follows from Isaacs and Rawlins' original insight. We focus on the generalization of Kaufmann's (2000) notion of 'conclude', i.e., $\sigma\left[\sigma^{\prime} \vdash \varphi\right]$ ('learning in a state $\sigma$ that a state $\sigma^{\prime}$ supports $\varphi$ ') mentioned in Remark 9. For structured contexts, we may define the operation of 'learning in a context $C$ that a context $C^{\prime}$ supports $\gamma^{\prime}$ (notation: $C\left[C^{\prime} \vdash \gamma\right]$ ) as follows (Isaacs \& Rawlins 2008: 293):

$C\left[C^{\prime} \vdash \varphi\right]:=\left\{\langle w, v\rangle \in C \mid \exists z \in W .\left(\langle w, z\rangle \in C^{\prime}\right.\right.$ or $\left.\langle z, v\rangle \in C^{\prime}\right)$ implies $\left.\langle w, v\rangle \in C^{\prime}[\gamma]\right\}$.

Let us substitute $C^{\prime}$ in $C\left[C^{\prime} \vdash \gamma\right]$ with $C[\varphi$ ! ], similarly to Remark 9. Then, we obtain Isaacs and Rawlins' semantics of conditionals in terms of structured contexts:

$C[\varphi ! \rightarrow \gamma]=\{\langle w, v\rangle \in C \mid \exists z \in W .(\langle w, z\rangle \in C[\varphi !]$ or $\langle z, v\rangle \in C[\varphi !])$ implies $\langle w, v\rangle \in C[\varphi !][\gamma]\}$

Since $\langle w, z\rangle \in C[\varphi !]$ and $\langle z, v\rangle \in C[\varphi !]$ are equivalent with $w(\varphi)=1$ and $v(\varphi)=1$ respectively, we may rewrite $C[\varphi ! \rightarrow \gamma]$ into:

$$
\{\langle w, v\rangle \in C \mid(w(\varphi)=1 \text { or } v(\varphi)=1) \text { implies }\langle w, v\rangle \in C[\varphi !][\gamma]\}
$$


Moreover, since $(w(\varphi)=1$ or $v(\varphi)=1)$ is equivalent with $\langle w, v\rangle \notin C[\neg \varphi !]$, our simplified semantics of conditionals is successfully derived from Isaacs and Rawlins' stack-based semantics: ${ }^{7}$

$$
C[\varphi ! \rightarrow \gamma]=\{\langle w, v\rangle \in C \mid\langle w, v\rangle \in C[\neg \varphi !] \text { or }\langle w, v\rangle \in C[\varphi !][\gamma]\}
$$

Note that both of $C[\varphi ! \rightarrow \psi !]$ and $C[\varphi ! \rightarrow \psi ?]$ are also structured contexts. ${ }^{8}$

Let us define the notion of support and consistency also for structured contexts.

$$
\begin{aligned}
& C \text { supports } \alpha \text { (written: } C=\alpha \text { ) if } C=C[\alpha] \text {. } \\
& \alpha \text { is consistent in } C \text { if } C[\alpha] \neq \emptyset .
\end{aligned}
$$

For instance, since we know that $C_{2} \neq C_{2}\left[q\right.$ ?] in Figure 3, $C_{2}$ does not support the interrogative $q$ ?, while updating $C_{2}[p !]$ with $q$ ? does not change the partition, thus $C_{2}[p !]$ supports $q$ ?. We note that, given a non-empty structured context $C$, any update of $C$ by a question $\varphi$ ? is always consistent in $C$.

As in Kaufmann 2000, we also obtain the following monotonicity result.

Proposition 12. $C[\alpha] \subseteq C$ for all contexts $C$ and query-formulas $\alpha \in \mathscr{Q} \mathscr{L}$.

What is the $d$-independent condition in this setting? First, let us consider the independence condition between declarative sentences. One might attempt to replace an information state $\sigma$ in Definition 5 with a structured context $C$ to obtain a possible candidate for the independence condition over structured contexts. However, this is insufficient, because $C[\neg \varphi !]=\emptyset$ is no longer equivalent to $C[\varphi !]=C$. ${ }^{9}$ That is, now that we have structured contexts, the candidate condition cannot be rewritten in terms of the notion of support. However, we can still preserve our previous intuition of independence in dynamic semantics: a query-formula $\psi$ ! is independent of $\varphi$ ! in $C$ if updating $C$ with $\varphi$ ! or $\neg \varphi$ ! does not affect the supportedness and the consistency of $\psi$ !. Thus, we provide Definition 13 as the notion of independence for structured contexts. From the condition (ii) of Definition 13, we can obtain the desired consequent entailment, as is the case with Theorem 1.

7 We owe this simplification of semantics of conditionals in terms of structured contexts to Makoto Kanazawa (p.c.).

8 Furthermore, perhaps surprisingly, $C[p ! \rightarrow q !]$ outputs an inquisitive context, although $p ! \rightarrow q$ ! is a declarative update. Let $W=\left\{w_{11}, w_{10}, w_{01}, w_{00}\right\}$ and let us take $C_{0}=W \times W$ from Figure 2 , i.e., an ignorant and indifferent structured context. If we update $C_{0}$ with $p ! \rightarrow q$ !, $C[p ! \rightarrow q$ ! $]$ $=C_{0}[\neg p !] \cup C_{0}[p !][q !]$ becomes an equivalence relation on $\left\{w_{11}, w_{01}, w_{00}\right\}$ where $\left\{w_{01}, w_{00}\right\}$ and $\left\{w_{11}\right\}$ form equivalence classes. Thus, $C[p ! \rightarrow q !]$ outputs an inquisitive context.

9 This is because for structured contexts, we cannot maintain the same semantics of negation as information states. That is, $C[\neg \varphi$ ! ] cannot be defined as $C \backslash C[\varphi !]:\langle w, v\rangle \in C[\neg \varphi !]$ iff $w(\varphi)=0$ and $v(\varphi)=0$. On the other hand, $\langle w, v\rangle \in C \backslash C[\varphi$ !] iff $w(\varphi)=0 \operatorname{or} v(\varphi)=0$. Therefore, there is a critical difference between 'and' and 'or'. 
Definition 13. $\psi$ ! is $d$-independent of $\varphi$ ! in $C$ if, given $\alpha$ 's consistency in $C$,

(i) $C[\beta] \neq \emptyset$ iff $C[\alpha][\beta] \neq \emptyset$ and

(ii) $C \models \beta$ iff $C[\alpha] \models \beta$,

for all $\alpha \in\{\varphi !, \neg \varphi !\}$ and all $\beta \in\{\psi !, \neg \psi !\}$.

Theorem 2. Let $\psi$ ! be $d$-independent of $\varphi$ ! in $C$ and $\varphi$ ! be consistent in $C$. Then, $C \models \varphi$ ! $\rightarrow \psi$ ! implies $C \models \psi$ !.

Proof. Assume that $C \models \varphi$ ! $\rightarrow \psi$ !. That is, $C[\neg \varphi !] \cup C[\varphi !][\psi !]=C$. We show that $C[\psi !]=C$. By $d$-independence, it suffices to show $C[\varphi !][\psi !]=C[\varphi !]$, i.e., $C[\varphi !] \subseteq C[\varphi !][\psi !]$ by Proposition 12. Fix any $\langle w, v\rangle \in C[\varphi !]$. We show that $\langle w, v\rangle \in$ $C[\varphi !][\psi !]$. Again by Proposition 12, we note $\langle w, v\rangle \in C$. So, $w(\varphi)=1$ and $v(\varphi)$ $=1$. This implies that $\langle w, v\rangle \notin C[\neg \varphi !]$. By $C[\neg \varphi !] \cup C[\varphi !][\psi !]=C$, we conclude $\langle w, v\rangle \in C[\varphi !][\psi !]$.

Now let us move to the independence of an interrogative $\psi$ ? from a declarative $\varphi$ ! over structured contexts. Basically, we can follow the same idea as in Definition 13 for declarative sentences, but we may simplify the statement of the definition. First of all, there is no need for us to consider both the case of $\beta$ is $\psi$ ? and the case of $\beta$ is $\neg \psi$ ? in Definition 13, since $C[\psi$ ? $]=C[\neg \psi$ ? ] for all structured contexts $C$. Second, the statement corresponding to (i) of Definition 13 always holds under the assumption of $\alpha$ 's consistency in $C$, and so the condition would be redundant for interrogatives. This is because the updates by interrogatives are always meaningful as we have already mentioned above. In particular, when we assume that $\alpha$ is consistent in $C$, we have $C \neq \emptyset$ and $C[\alpha] \neq \emptyset$, hence $C[\psi ?] \neq \emptyset$ and $C[\alpha][\psi ?] \neq \emptyset$.

Definition 14. $\psi$ ? is $d$-independent of $\varphi$ ! in $C$ if, given $\alpha$ 's consistency in $C$,

$$
C \models \psi ? \quad \text { iff } \quad C[\alpha] \models \psi ?
$$

for all $\alpha \in\{\varphi !, \neg \varphi !\}$.

To illustrate, let us consider the following examples:

a. $q$ ? is $d$-independent from $p$ ! in $C_{1}$ of Figure 4 . This is because an update by $q$ ? gives us a finer partition which allows us to distinguish between $q$ worlds and $\neg q$-worlds, but all three structured contexts in Figure 4 already distinguish those two 'zones'. Let us see why via definition. When $\alpha$ is $p$ !, it suffices to show the equivalence between $C_{1} \models q$ ? and $C_{1}[p$ ! $] \models q$ ?, but both of them hold as in Figure 4. Similarly, for the case where $\alpha$ is $\neg p$ !, it is easy to see the equivalence between $C_{1} \models q$ ? and $C_{1}[\neg p !] \models q$ ? 
Conditional independence and biscuit conditional questions

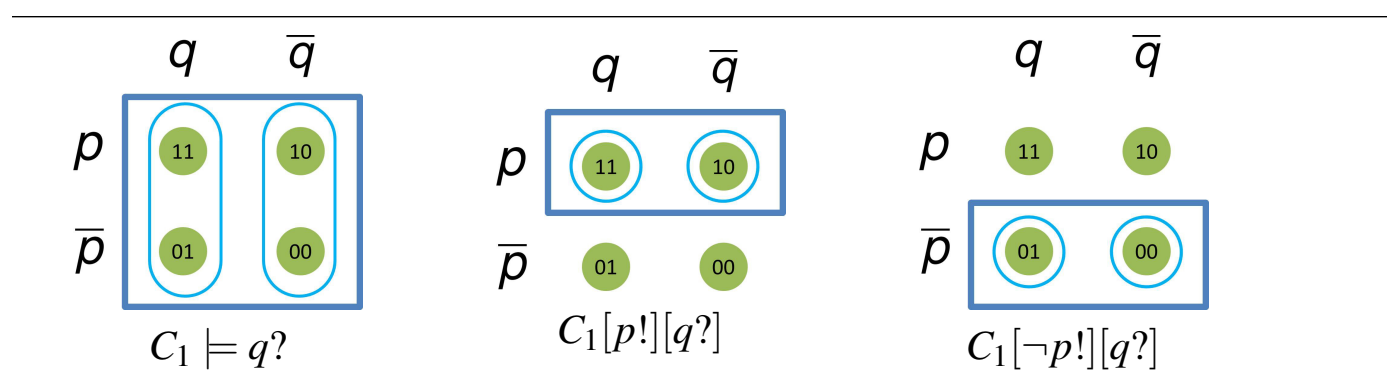

Figure $4 q$ ? is $d$-independent from $p$ ! in $C_{1}$

b. $q$ ? is not $d$-independent from $p$ ! in $C_{2}$ of Figure 3. Recall that the structured context $C_{2}$ does not support $q$ ?, while the updated context $C_{2}[p !]$ by the declarative $p$ ! support $q$ ?

Then, by an argument similar to Theorem 2, we can establish the following:

Theorem 3. Let $\psi$ ? be $d$-independent of $\varphi$ ! in $C$ and $\varphi$ ! be consistent in $C$. Then, $C \models \varphi$ ! $\rightarrow \psi$ ? implies $C \models \psi$ ?

Let us take (1a) and (4) as examples. Assume a similar non-magical situation where the speaker being thirsty does not determine the presence/absence of drinks in the fridge. Given the $d$-independence condition and Theorem 2, both the consequent declarative 'there's beer in the fridge' and the consequent interrogative 'is there anything in the fridge?' are supported. Thus, our condition derives the consequent entailment for both biscuit conditional statements and questions in the dynamic framework.

\section{Conclusion}

\subsection{Summary}

We develop a dynamic and non-symmetric version of independence tailored for both information states and structured contexts. Franke's proposal is further supported in that there is no need for stipulating special semantics for biscuit conditionals, since the "feeling of entailment" of biscuit conditional questions as well as statements can be derived from the existing dynamic semantics of conditionals and our dynamic independence.

\subsection{Future directions}

There are several future directions for research related to this analysis of biscuit conditionals and the notion of independence. First, the current paper employed 
Groenedijk's (1999) structured context to characterize conditional questions in truthconditional semantics. However, inquisitive semantics developed by Groenendijk and his colleagues (Groenendijk \& Roelofsen 2009, among others) enables us to define the semantics of interrogative sentences in terms of an (unstructured) information state. In inquisitive semantics, the meaning of a sentence is obtained by support-conditions. An information state supports an interrogative sentence ? $p$, which is equivalent to $p \vee \neg p$, if it supports $p$ or $\neg p$. Thus, it appears to be fruitful to investigate whether our notion of independence can be extended to inquisitive semantics. Furthermore, in a recent version of the framework called "Suppositional Inquisitive Semantics" (Groenendijk \& Roelofsen 2013), there is a semantic relation called dismissing a supposition, which characterizes Isaacs and Rawlins' (2008) intuition of 'denial of the antecedent', as mentioned in section 3.1.

Second, we restricted our syntax to the propositional level in this paper. Since there are first-order extensions of dynamic semantics, it would be interesting to see whether it is possible to extend our idea and analysis to the first-order case where the property of monotonicity of updates is maintained.

Third, in this paper, we extracted the semantics of conditional declaratives and interrogatives on structured contexts from the stack model of Isaacs \& Rawlins (2008) and defined the notion of $d$-independence. Alternatively, we may generalize our idea of independence to Isaacs and Rawlins' (2008) original stack model.

\section{References}

Franke, Michael. 2007. The pragmatics of biscuit conditionals. In Maria Aloni, Paul Dekker \& Floris Roelofson (eds.), 16th Amsterdam Colloquium, 91-96. Universiteit van Amsterdam.

Franke, Michael. 2009. Signal to Act: Game Theory in Pragmatics: Universiteit van Amsterdam $\mathrm{PhD}$ dissertation.

Groenendijk, Jeroen. 1999. The logic of interrogation. In Tanya Matthews \& Devon Strolovitch (eds.), Semantics and Linguistic Theory (SALT) 9, 109-126. Ithaca, NY: CLC Publications.

Groenendijk, Jeroen \& Floris Roelofsen. 2009. Inquisitive semantics and pragmatics. Workshop on Language, Communication, and Rational Agency at Stanford, May 30-31, 2009.

Groenendijk, Jeroen \& Floris Roelofsen. 2013. Suppositional inquisitive semantics. Workshop on Inquisitive Logic and Dependence Logic, ILLC, Amsterdam, June 17, 2013.

Hamblin, C.L. 1958. Questions. Australasian Journal of Philosophy 36. 159-168.

Heim, Irene. 1982. The semantics of definite and indefinite noun phrases: University of Massachussets, Amherst PhD dissertation. 
Conditional independence and biscuit conditional questions

Iatridou, Sabine. 1991. Topics in conditionals. Cambridge, MA: MIT PhD dissertation.

Isaacs, James \& Kyle Rawlins. 2008. Conditional questions. Journal of Semantics 25. 269-319.

Karttunen, Lauri. 1974. Presupposition and linguistic context. Theoretical Linguistics 1(1/2). 182-194.

Kaufmann, Stefan. 2000. Dynamic context management. In Martina Faller, Stefan Kaufmann \& Marc Pauly (eds.), Formalizing the Dynamics of Information, 171-188. Stanford, CA: CSLI.

Kratzer, Angelika. 1981. The notional category of modality. In Hans-Jürgen Eikmeyer \& Hannes Rieser (eds.), Words, Worlds, and Contexts, 38-74. Berlin: Walter de Gruyter.

Kratzer, Angelika. 1991. Modality. In Arnim von Stechow \& Dieter Wunderlich (eds.), Semantics: An iternational handbook of contemporary research, 639-650. Berlin: de Gruyter.

Lewis, David. 1988. Relevant impliation. Theoria 54. 161-237.

Ramsey, Frank Plumpton. 1931. General propositions and causality. In Richard Bevan Braithwaite (ed.), The Foundations of Mathematics: Collected Papers of Frank P. Ramsey, 237-255. London: Routledge.

van Rooij, Robert. 2007. Strengthening conditional presuppositions. Journal of Semantics 24. 289-304.

Siegel, Muffy. 2006. Biscuit conditionals: Quantification over potential literal acts. Linguistics and Philosophy 29(2). 167 - 203.

Stalnaker, Robert. 1968. A theory of conditionals. In Nicholas Resher (ed.), Studies in Logical Theory, Oxford: Blackwell.

Velissaratou, Sophia. 2000. Conditional questions and which-interrogatives. University of Amsterdam MA thesis.

Veltman, Frank. 1996. Defaults in update semantics. Journal of Philosophical Logic 25(3). 221-261.

Katsuhiko Sano

School of Information Science

Japan Advanced Institute of Science and Technology

1-1 Asahidai, Nomi

Ishikawa, Japan

v-sano@jaist.ac.jp
Yurie Hara

Department of Linguistics and Translation

City University of Hong Kong

81 Tat Chee Avenue

Kowloon, Hong Kong SAR

y.hara@ cityu.edu.hk 